\begin{tabular}{|c|c|}
\hline Citation & $\begin{array}{l}\text { Bing Li, Josep Puigmartí-Luis, Alain M. Jonas, David B. Amabilino and Steven } \\
\text { De Feyter } \\
\text { Hierarchical growth of curved organic nanowires upon evaporation } \\
\text { induced self-assembly } \\
\text { Chemical Communications, } 2014,50,13216 \text { - } 13219\end{array}$ \\
\hline Archived version & $\begin{array}{l}\text { Author manuscript: the content is identical to the content of the published } \\
\text { paper, but without the final typesetting by the publisher }\end{array}$ \\
\hline Published version & $\begin{array}{l}\text { insert link to the published version of your paper } \\
\text { http://dx.doi.org/10.1039/c4cc03676j }\end{array}$ \\
\hline Journal homepage & $\begin{array}{l}\text { insert link to the journal homepage of your paper } \\
\text { http://pubs.rsc.org/en/journals/journal/cc }\end{array}$ \\
\hline Author contact & $\begin{array}{l}\text { your email steven.defeyter@kuleuven.be } \\
\text { your phone number + } 32(0) 16327921\end{array}$ \\
\hline IR & url in Lirias https://lirias.kuleuven.be/handle/123456789/477344 \\
\hline
\end{tabular}

(article begins on next page) 


\title{
Hierarchical growth of curved organic nanowires upon evaporation induced self-assembly
}

\author{
Bing Li, ${ }^{a \#}$ Josep Puigmartí-Luis, ${ }^{b \#}$ Alain M. Jonas ${ }^{c}$, David B. Amabilino ${ }^{b^{*}}$ and Steven De Feyter ${ }^{a^{*}}$ \\ Received (in $X X X, X X X) X t h X X X X X X X X X 20 X X$, Accepted $X$ th $X X X X X X X X X 20 X X$ \\ 5 DOI: 10.1039/b000000x
}

Self-assembly of a pi-electron rich molecule capable of forming self-assembled monolayers at the surface of graphite displays hierarchical growth of multilayers and complex curved nanostructures including nanospirals and concentric 10 nanorings upon evaporation of a solution.

The functionality of $\pi$-electron systems often depends critically upon the superstructure and the interface where they form. ${ }^{1}$ Many different types of morphologies have been reported among which fibres and tapes are the most common. ${ }^{2}$ One example of the 15 formation of functional supramolecular rings - which subsequently stack to form tubes - is the self-assembly of a $C_{2}$ symmetrical topologically linear acceptor-donor-acceptor bolaamphiphile. ${ }^{3}$ Occasionally, amphiphilic compounds with no particular electronic functionality have displayed remarkable curved 20 morphologies. For example, Liu and co-workers described the formation of spirals of a barbituric acid derivative at the air/water interface, ${ }^{4}$ whereas, Wan et al. demonstrated the formation of similar spiral like superstructures upon controlled evaporation of a specific solvent mixture containing saturated fatty acids. ${ }^{5}$

25 We have studied the assembly of monolayers and multilayers of molecules which contain tetrathiafulvalenes (TTFs) and have shown that depending on the number and position of amide groups which assist in the intermolecular attraction various fibre-like morphologies are obtained. ${ }^{6}$ One of these compounds (1), the 30 object of this study, forms physisorbed multilayers at the interface between graphite and octanoic acid in a dynamic system where the liquid is still present. ${ }^{7}$ However, from the viewpoint of making solid material with unusual topographies and whose proerties might be studied, it is interesting to be able to form superstructures 35 upon evaporation of the solvent.

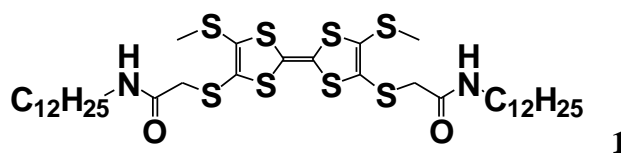
1

Compound 1 forms short fibres on graphite when crystallized 40 from evaporating chloroform. ${ }^{8}$ A present objective of ours is to improve the morphology of this compound. Here we report the results of evaporation of octanoic acid solutions of $\mathbf{1}$ on graphite.

When a solution of $\mathbf{1}$ in octanoic acid was allowed to evaporate on the basal plane of a freshly-cleaved highly oriented pyrolytic
45 graphite (HOPG), atomic force microscope (AFM) imaging reveals that monolayers and multilayers of the material are formed (images not shown) where straight fibres are formed in domains, rather like the physisorbed layers that we described at the liquid/solid interface. ${ }^{7}$ However, domains of disc-like aggregates 50 with holes at their centres form mainly hexagonal close packed assemblies (Fig. 1; see supporting information for experimental details) in certain areas. The discs (which are a little under $50 \mathrm{~nm}$ in diameter on average; Fig. S1) are formed of concentric homogeneous curved layers of lines of molecule $\mathbf{1}$. Some of the 55 objects are comprised of concentric circles, others are Archimedean spirals (a spiral comprised of one strand with equal spacing between the lines at each turn) and others are similar to Fermat's spiral (two strands winding around emerging from a centre, where they are joined in that object but not in the objects 60 observed here). There are also more complex objects where three (or more) strands form a spiral. Both clockwise and anticlockwise spirals are observed.

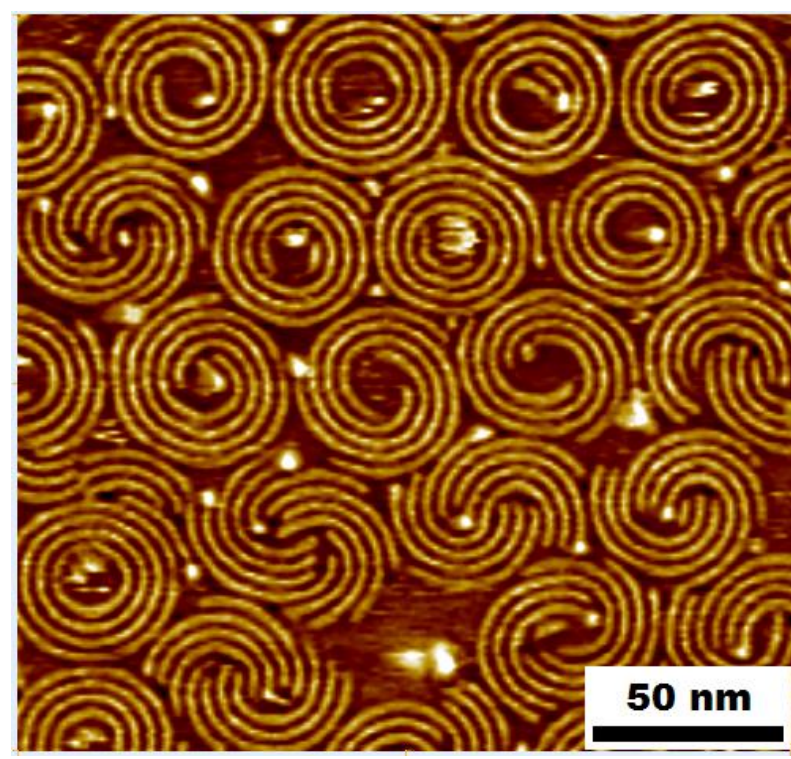

Fig. 1: A representative AFM phase image of a film of $\mathbf{1}$ on freshly65 cleaved HOPG, formed upon evaporation of the solvent, 1-octanoic acid. Various topologies are formed, including disklike features composed of concentric rings and spirals.

Often, at the ends of the strands either in the centre of the discs or on the outer edges, additional residues can be found. We can rule 
out that the debris in the centre of the circular features is a nucleation centre because this mechanism would not give rise to this kind of size-limited structure, and the debris-free discs are also frequently observed. It seems, rather, that strands are formed, and 5 then, either in isolation or by combining between them, come together to form the disc-shaped objects.

The concentric rings and spirals sometimes lay on top of a monolayer of 1 (Fig. 2). However, such features were also found on bare HOPG surface (Fig. 3A and 3B) or surrounded by thicker 10 layers of $\mathbf{1}$ (Fig. 3C and 3D). In addition, those disc-like aggregates show preference to appear at graphite step edges (Fig. S2), perhaps because of solvent pinning before complete evaporation of the drop. These observations suggest that the formation of the discs is neither induced by the HOPG lattice nor templated by the lamella 15 monolayer of $\mathbf{1}$. Most likely, $\mathbf{1}$ has formed strands and assembled into disc-like structures prior to adsorbing on HOPG. The higher surface energy of graphite step edges most probably leads to the frequent appearance of the disc-like structures at these sites.

Under other conditions the spirals are not observed: The results 20 on gold, mica and silicon differ significantly from those on HOPG (Fig. S3). Only aggregated particles or clean surfaces were observed on hydrophilic gold and mica. Visualisation of any (supra) molecular features on bare $\mathrm{Si}$ is a challenging task due to adverse effects of the rough and hard surface on the AFM imaging. When 25 deposited from chlorofrom or toluene, only the wire-like structures are observed. ${ }^{8}$

Deposition of a thick film 1 on HOPG results in both disc-like features and bundled-wire structures (Fig. S4A and S4B). Even pure wire-type phases were occasionally found (Fig. S4C and S4D).

30 Those observations highlight that $\mathbf{1}$ is capable of self-assembling into strands/wires and able to form disc-like structures under certain conditions. The formation of bundles and aggregates of wires is probably caused by the higher concentration of strands near complete solvent evaporation.

35 The height of the disc-like structures is approximately $6 \AA$ (Fig. 2 ). The dark troughs in between concentric fibres are presumably occupied by the alkyl chains of $\mathbf{1}$. The spacing between fibres is $4.1 \pm 0.3 \mathrm{~nm}$, which is smaller than the length of an extended molecule of $1(4.5 \mathrm{~nm})$, and also somewhat less than the distance 40 between two TTF rows in the lamella monolayer $(4.6 \pm 0.2 \mathrm{~nm}$; Fig. 2, 4A and 4B).

This implies that the molecular geometry in the disc-type features is different from the one that is normally observed in lamella-type monolayers on HOPG, ${ }^{6,7}$ a suggestion backed up by 45 the very different phase contrast that they display in the AFM images (Fig. 3B). Furthermore, the high curvature of the fibres in these disc-type structures does not match the expected architecture of physisorbed self-assembled monolayers of alkylated molecules templated by the crystalline lattice of HOPG. This further supports 50 our view that the disc-type features are formed prior to adsorption on HOPG.

We suggest the following tentative and speculative mechanism. Hydrogen bonds and intermolecular S-S interactions are strong enough to direct the self-assembly of the TTF derivatives into 55 supramolecular wires in appropriate solution conditions.
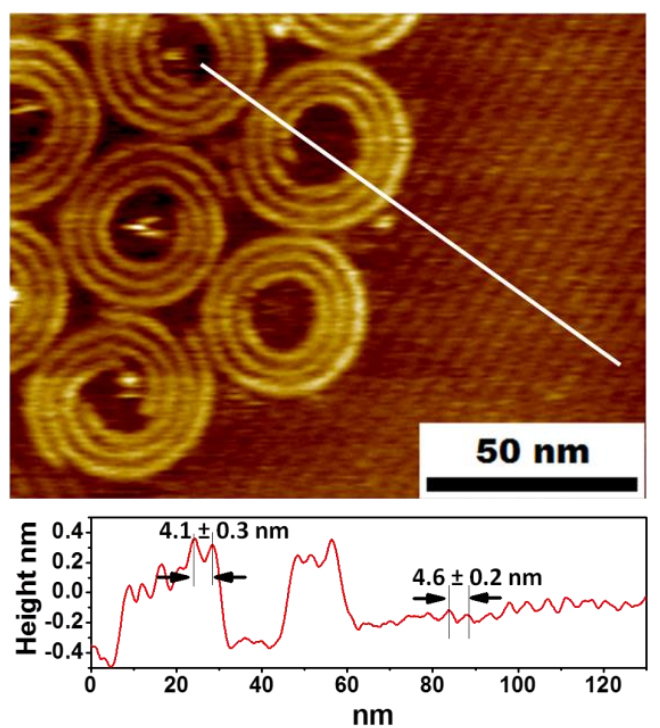

Fig. 2: AFM image showing some disc-like objects formed by $\mathbf{1}$ on top of a layer of the compound where the parallel straight fibre polymorph can be observed. Below the image is a contour corresponding to the line in the 60 image and showing the height of the disc-like feature, and the common uniform spacing.

In absence of forces that constrain the alkyl chains to adopt an extended all-trans conformation - the latter situation is typically observed on HOPG - alternative alkyl chain conformations might ${ }_{65}$ be adopted when lowering the total energy of the system (Fig. 4C$4 \mathrm{~F})$. This explains the shorter spacing of the lines in disc-type structures as compared to those of the lamella stripes in the monolayer. In addition, solvent molecules of octanoic acid may participate in the assembly of molecular strands via van der Waals 70 interactions between alkyl chains of octanoic acid and $\mathbf{1}$, and via hydrogen bonding. If octanoic acid molecules incorporate at one side of the molecular wire, the interspacing between alkyl chains of adjacent molecules of $\mathbf{1}$ will increase to some extent (as indicated by arrows in Fig. 4E and 4F) and lead to the bending of 75 the molecular wire, eventually leading to the formation of these interesting spirals or concentric circles. Given the achiral nature of octanoic acid, such mechanism must imply dissymmetric packing, the details of which remain to be explored.

The inner most circles are rather consistent in size, i.e. ca. 20 $80 \mathrm{~nm}$. The reason for this lower-limit could be steric hindrance, limit to curvature. There is also a limitation in the maximum diameter of the outer most circle $(\sim 45-50 \mathrm{~nm}$ (Fig. S1)), i.e. concentric circles going beyond four "layers" were never observed..If more than four "layers", the wires may tend to form straight stripes as 85 the interactions between octanoic acid and molecular wires of $\mathbf{1}$ become small.

The fact that more spirals are observed at step edges is probably due to the pinning of the dewetting receding line at these locations, possibly as the result of the formation of droplets. If the 90 destabilization is governed by van der Waals forces beteren alkyl chains of solvent and $\mathbf{1}$ and the surface, the process should be dependent on the nature of the solvent (its "polarity"), and on the nature of the substrate. This fact could explain the lack of spirals from other solvents and upon evaporation from octanoic acid on 95 other substrates. If the destabilization were due to structural forces, they should appear on any substrate. 


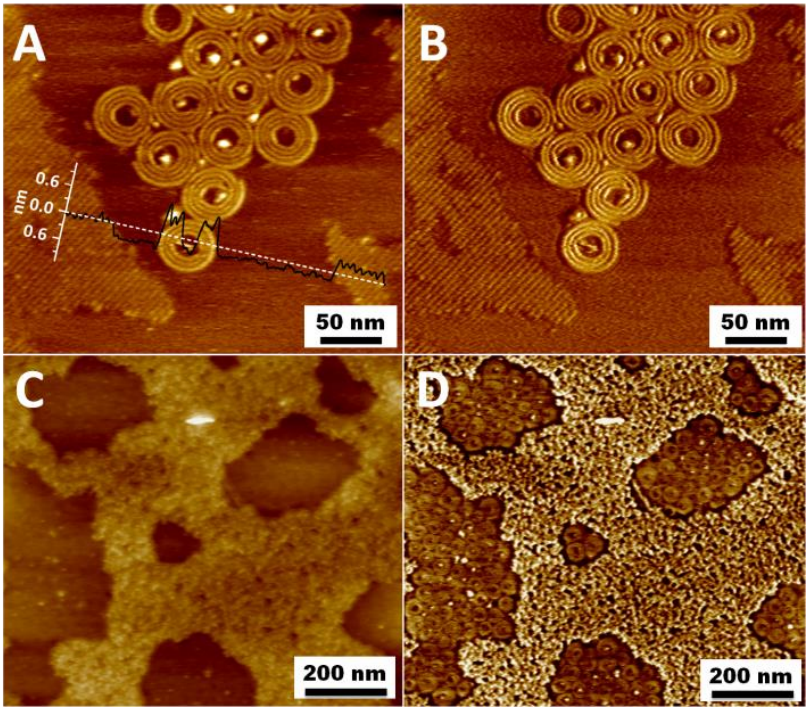

Fig. 3: (A) and (B): AFM height image and its corresponding phase image (where contrast makes the discs easier to discern) showing the disc-like objects of 1 formed on bare HOPG surface. (C) and (D): AFM height image 5 and its corresponding phase image showing the disc-like objects in the voids of thicker layers of $\mathbf{1}$.

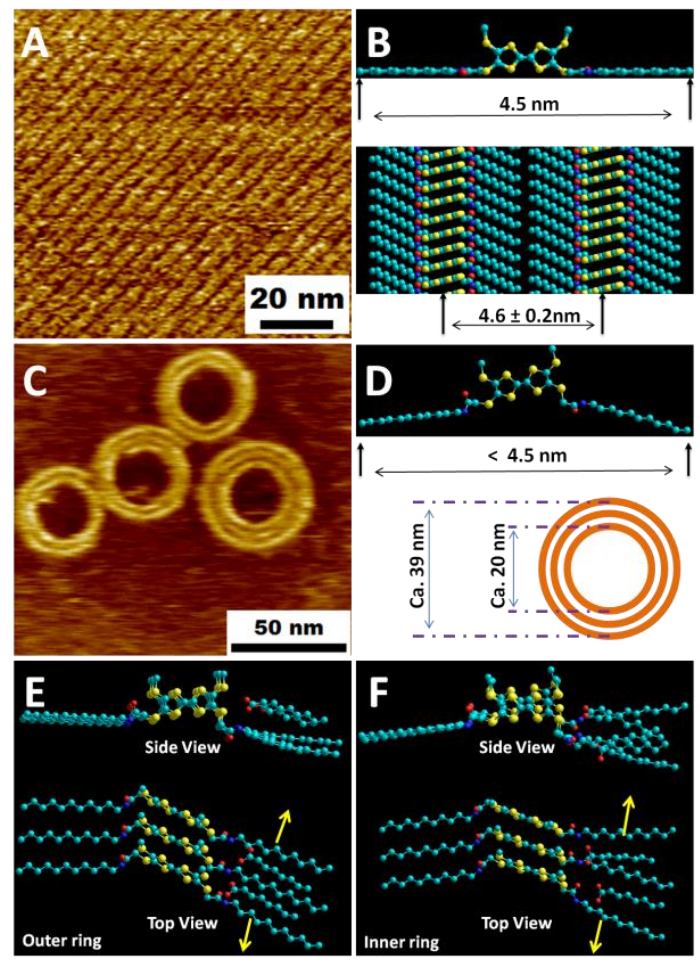

Fig. 4: (A) A typical phase image of lamellar pattern formed by 1 on HOPG and (B) its corresponding molecular geometry and packing model. 10 (C) A typical phase image of disc structure formed by 1 on HOPG and (B) a proposed geometry of 1 in the disc and a schematic showing a concentric circle model consisting of 3 ring layers. (E) and (F) molecular model segments of the outer ring and inner ring in the disc structure, respectively. Another possibility is the one where the TTF is at right 15 angles to the plane of the surface

However, the circular structures could form at the solvent-air interface and then assemble as the drop is evaporating at the step edge. If the droplet were to break earlier (as the result of destabilisation of the fluid film because of varying interactions
20 with the substrate), the concentration of $\mathbf{1}$ would not be sufficient to allow the formation of these circular structures. It is not a classic "coffee-stain" mark, because the rings are extremely small and are clustered together. The effect is thus unique and curious.

In conclusion, we have demonstrated the formation of unique 25 supramolecular concentric rings and spirals formed by a TTF derivative upon very specific solvent evaporation on a specific surface. These spiral structures have only been observed upon evaporation of octanoic acid solutions on graphite, suggesting that the solvent interaction with the solute and the interaction of this 30 assembly with the graphite plays a defining role in the superstructures that are formed through non-covalent bonds at highly concentrated conditions. Future studies will aim at unravelling the precise growth mechanism of these exotic structures.

This work was supported by the Fund of Scientific ResearchFlanders (FWO), KU Leuven (GOA) and the Belgian Federal Science Policy Office (IAP-7/05). The research leading to these results has received funding from the Generalitat de Catalunya 40 (2009 SGR 158) and the MINECO (CTQ2010-16339). JPL thanks the MINECO for a Ramo'n y Cajal contract (RYC-2011-08071). This research has also received funding from the European Research Council under the European Union's Seventh Framework Programme (FP7/2007-2013)/ERC Grant Agreement ${ }_{45}$ No. 340324.

\section{Notes and references}

$\begin{array}{cccccr}{ }^{a} \text { KU Leuven, Celestijnenlaan } & \text { 200F, } 3001 \text { Heverlee, Belgium. Fax: }+32 \\ 1632 & 7990 ; \quad \text { Tel: } \quad+32 & 1632 & 7921 ; & \text { E-mail: }\end{array}$ steven.defeyter@chem.kuleuven.be

${ }_{50}{ }^{b}$ Institut de Ciència de Materials de Barcelona (ICMAB-CSIC), Campus Universitari, 08193 Bellaterra,Catalonia, Spain. Fax: +34 935805 729; Tel: +34935801 853; E-mail: amabilino@icmab.es c Institute of Condensed Matter and Nanosciences, Université catholique de Louvain, Croix du Sud 1/L7.04.02, 1348 Louvain-la-Neuve (Belgium).

$55{ }^{\#}$ Present address: Institute of Materials Research and Engineering, A*STAR (Agency for Science, Technology and Research), 3 Research Link, Singapore 117602

\#\# Present address: Empa, Laboratory for Protection and Physiology, Lerchenfeldstrasse 5, 9014 St. Gallen, Switzerland.

1 a) H. Sirringhaus, P. J. Brown, R. H. Friend, M. M. Nielsen, K Bechgaard, B. M. W. Langeveld-Voss, A. J. H. Spiering, R. A. J. Janssen, E. W. Meijer, P. Herwig, and D. M. de Leeuw, Nature, 1999, 401, 685. b) A. Miura, P. Jonkheijm, S. De Feyter, A. Schenning, E.

65 W. Meijer, and F. C. De Schryver, Small, 2005, 1, 131. c) W. H. Lee, J. Park, S. H. Sim, S. Lim, K. S. Kim, B. H. Hong, and K. Cho, J. Am. Chem. Soc., 2011, 133, 4447.

2 a) A. J. J. V. Heyen, C. C. Buron, Q. Tianshi, R. Bauer, A. M. Jonas, K. Muellen, F. C. De Schryver, and S. De Feyter, Small, 2008, 4, 1160

70 b) R. Matmour, I. De Cat, S. J. George, W. Adriaens, P. Leclere, P. H. H. Bomans, N. A. J. M. Sommerdijk, J. C. Gielen, P. C. M. Christianen, J. T. Heldens, J. C. M. van Hest, D. W. P. M. Lowik, S De Feyter, E. W. Meijer, and A. P. H. J. Schenning, J. Am. Chem. Soc., 2008, 130, 14576. c) M. Wang, A. R. Mohebbi, Y. Sun, and F. Wudl, Angew. Chem., Int. Ed., 2012, 51, 6920.

3 S. Tu, S.H. Kim, J. Joseph, D.A. Modarelli and J.R. Parquette, J. Am. Chem. Soc., 2011, 133, 19125.

4 X. Huang, C. Li, S. G. Jiang, X. S. Wang, B. W. Zhang and M. H. Liu, J. Am. Chem. Soc., 2004, 126, 1322.

805 L. Wan, L. Li and G. Mao, Nanoscale Res. Lett., 2011, 6, 49

6 a) J. Puigmartí-Luis, A. Minoia, H. Uji-i, C. Rovira, J. Cornil, S. De Feyter, R. Lazzaroni and D.B. Amabilino, J. Am. Chem. Soc., 2006, 
128, 12602. b) S. Lei, J. Puigmartí-Luis, A. Minoia, M. Van der Auweraer, C. Rovira, R. Lazzaroni, D. B. Amabilino and S. De Feyter, Chem. Commun., 2008, 703. c) C. Munuera, J. Puigmartí-Luis, M. Paradinas, L. Garzón, D. B. Amabilino and C. Ocal, Small, 2009, 5, 214.

7 J. Puigmartí-Luis, A. Minoia, S. Lei, V. Geskin, B. Li, R. Lazzaroni, S. De Feyter, D. B. Amabilino, Chem. Sci., 2011, $2,1945$.

8 J. Puigmartí-Luis, A. Minoia, A. Pérez del Pino, G. Ujaque, C. Rovira, A. Lledós, R. Lazzaroni and D. B. Amabilino, Chem. Eur. J., 2006, 12, 9161. 